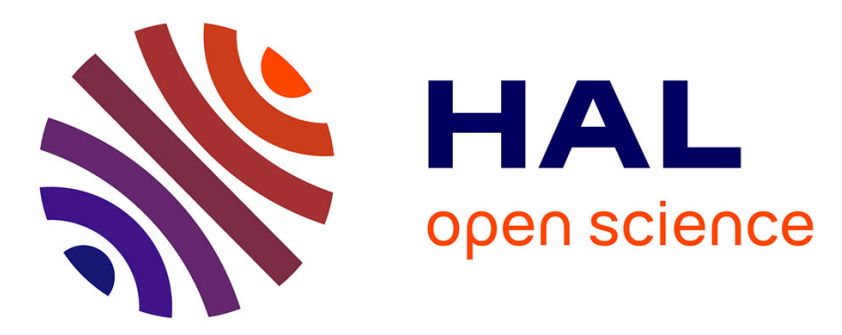

\title{
Morphology study of femtosecond laser nano-structured borosilicate glass using atomic force microscopy and scanning electron microscopy
}

\author{
B. Delobelle, F. Courvoisier, P. Delobelle
}

\section{- To cite this version:}

B. Delobelle, F. Courvoisier, P. Delobelle. Morphology study of femtosecond laser nano-structured borosilicate glass using atomic force microscopy and scanning electron microscopy. Optics and Lasers in Engineering, 2009, 48 (5), pp.616-625. 10.1016/j.optlaseng.2009.09.013 . hal-00453814

\author{
HAL Id: hal-00453814 \\ https://hal.science/hal-00453814
}

Submitted on 18 Apr 2021

HAL is a multi-disciplinary open access archive for the deposit and dissemination of scientific research documents, whether they are published or not. The documents may come from teaching and research institutions in France or abroad, or from public or private research centers.
L'archive ouverte pluridisciplinaire HAL, est destinée au dépôt et à la diffusion de documents scientifiques de niveau recherche, publiés ou non, émanant des établissements d'enseignement et de recherche français ou étrangers, des laboratoires publics ou privés.

\section{(c)(1)}

Distributed under a Creative Commons Attribution| 4.0 International License 


\title{
Morphology study of femtosecond laser nano-structured borosilicate glass using atomic force microscopy and scanning electron microscopy
}

\author{
Benoît Delobelle ${ }^{\mathrm{a}, \mathrm{b}}$, François Courvoisier ${ }^{\mathrm{b}}$, Patrick Delobelle ${ }^{\mathrm{a}, *}$ \\ a Department DMA, FEMTO-ST (UMR CNRS 6174), Université de Franche Comté, 24 chemin de l'Epitaphe, 25000 Besançon, France \\ b Department LOPMD, FEMTO-ST (UMR CNRS 6174), Université de Franche Comté, 16 route de Gray, 25030 Besançon Cedex, France
}

\begin{abstract}
The morphology of nano-craters drilled in borosilicate glass by single-shot femtosecond laser ablation has been studied by atomic force microscopy and scanning electron microscopy. The influence of polarization, numerical aperture (NA=0.4 and 0.8$)$ and fluence $\left(3<F<18 \mathrm{~J} \mathrm{~cm}^{-2}\right)$ was systematically investigated in the case of a strong geometrical confinement, leading to nanometric scale in all spatial dimensions. Indeed, the structure size is not restricted by the diffraction limit but determined by the laser pulse stability and the material properties.

The dimensions of the principal and of the secondary (self-focusing) craters, and of the rim have been studied in detail. Different relationships have been proposed for the evolutions of the depths and of the different diameters of the craters as functions of the position of the specimen surface through the beam-material interaction region, and of the characteristics of the laser.
\end{abstract}

\section{Introduction}

Ultrafast laser processing of materials continues to be an intense subject of research from fundamentals and modeling to a large field of applications [1,2]. Among them, the deterministic machining at submicrometer scale has attracted much attention [3-9] with applications to materials nano-structure for memories, micro-optical devices, mechanical modifications, sensors, wettability modifications, bio-integration, etc.

However, though a precisely defined threshold, ultrafast laser ablation results from a complex series of physical processes: multiphotons and tunnel ionization, avalanche ionization, carriercarrier scattering, carrier-phonon scattering, material phase change, shock wave emission, thermal diffusion, material ejection followed by condensation and eventually resolidification of clusters and melt material. As these processes have time scales that extend from femtoseconds to microseconds, the modeling is extremely difficult and has generated many theoretical developments from the ultrafast dynamics of free electron generation to non-equilibrium thermodynamics involving large-scale molecular dynamics simulations [9-13].

However, most of experimental and modeling results of the morphology of ultrafast laser nano-ablation only concern the case of very small aspect ratio holes. Indeed, surface profiling of nano-

\footnotetext{
* Corresponding author. Tel.: +33381666013; fax: +33381666700.

E-mail address: patrick.delobelle@univ-fcomte.fr (P. Delobelle).
}

holes has been performed on holes with a diameter on the micronic size because they could be interpreted in the light of 1D numerical models [9,11,14]. Englert et al. [15] have recently investigated the influence of temporal phase of femtosecond pulses on ablation efficiency of fused silica by means of atomic force microscopy (AFM) but did not relate to the morphology question. Chimmalgi et al. [5] obtained record nano-ablations down to $10 \mathrm{~nm}$ of depth by means of a scanning probe microscope coupled to a femtosecond laser. To the best of the author's knowledge, full surface profiling of single-shot femtosecond laser drilled nano-holes in the focal region has never been performed with a systematic approach in dielectrics. Indeed, the competition between multiphotons, tunnel and avalanche ionization effects, energy diffusion effects $[10,14]$ and geometrical confinement effects [16], thermocapillary forces (Marangoni effect) [17] on $3 \mathrm{D}$ nanometer scale render the morphology of the single-shot craters difficult to predict.

Here, we present the results of a systematic AFM and scanning electron microscopy (SEM) study through the focal region of nearthreshold single-shot femtosecond laser ablation nano-holes in borosilicate glass under ambient atmosphere. The purpose is to extract relevant information on the 3D morphology of nano-holes drilled in dielectrics for applications to the fields of sensors, integrated phononics, plasmonics and eventually photonics. Moreover, as this study concerns nanometric scales on three dimensions, it becomes in accordance with the accessible range of sizes for numerical simulations [16] and is intended to serve as a benchmark. 


\section{Experiments}

\subsection{Experimental setup}

The amplified laser source (Spitfire ProV, Spectra-physics) emits $120 \mathrm{fs}$ laser pulses with a central wavelength $\lambda$ of $800 \mathrm{~nm}$, at a repetition rate $f$ of $5 \mathrm{kHz}$. An independent Pockels cell system with a thin-film polarizer plays the role of an optical shutter that enables single-shot illuminations. An ensemble of neutral density filters allows adjusting the pulse energy. A polarizing cube and a zero-order quarter wave plate allow for the production of a circular polarization before the laser beam passes through a microscope objective (MO), focusing onto a glass plate. Two types of Olympus Plan-fluor infinitely-corrected microscope objectives were used: $\times 20$ with 0.4 numerical aperture (NA) and $\times 50$ with $\mathrm{NA}=0.8$. The beam diameter being larger than the entrance aperture of the MO, the Airy spot sizes are 2.4 and $1.2 \mu \mathrm{m}$.

The glass slides were mounted over a 3D positioning motorized stage (Newport ILS M-VP25) with bidirectional repeatability better than $200 \mathrm{~nm}$ and the sample planarity was controlled to be less than $1 \mathrm{mrad}$. The positioning of the samples was achieved by imaging on a CCD camera with depths of field of 2 and $0.5 \mu \mathrm{m}$ with the $\times 20 \mathrm{MO}$ and $\times 50 \mathrm{MO}$, respectively.

Specific care has been devoted to the cleanliness of the beam and the dispersion of all optics has been pre-compensated with the compressor of the chirped-pulse amplifier of the laser chain. The precompensation has been carefully adjusted by measuring the pulse duration $\tau$ with a GRENOUILLE after the laser beam has passed through the microscope objective and was collimated by a thin lens with negligible dispersion. The measured pulse duration $\tau$ is about $120 \mathrm{fs}$.

The transmitted mean laser power $P$ after the microscope objectives has been measured with a calibrated power-meter (GENTEC, XLP 12) whose measure range is $10 \mu \mathrm{W}<P<3 \mathrm{~W}$ corresponding to a pulse energy $E$ in the range $2 \times 10^{-3}$ $\mu \mathrm{J}<E<600 \mu \mathrm{J}$. Single-shot illuminations of the sample were performed under atmospheric conditions, for different pulse energies in the range of $1.6 \times 10^{-2}-0.6 \mu \mathrm{J}$, near the ablation threshold of fused silica. For each laser power, the sample was translated through the focal region, in the vertical direction $Z$, by steps of $250 \mathrm{~nm}$ over a range of $15 \mu \mathrm{m}$. After each laser shot, corresponding to a fixed $z$ value, the sample was translated in plane by $5 \mu \mathrm{m}$ in the $X$ direction. After eight shoots on the same line in the $X$ direction, a new line of eight shoots is realized with the same origin of the first one, but translated of $20 \mu \mathrm{m}$ in the $Y$ direction. Then, this sequence has been repeated ten times, covering the $Z$ focal region over $15 \mu \mathrm{m}$. This procedure allows a compact area of the silica plate to be nano-structured. To test the reproducibility of this method, six specimens (noted Spec. 1-6) with the same or different nano-structurations have been characterized.

\section{2. $A F M$ and SEM measurements}

Atomic force microscopy measurements have been performed on each illuminated zone in a non-contact mode, with a spatial resolution of $20 \mathrm{~nm}$ in the sample plane and with $5 \mathrm{~nm}$ in depth. The microscope is a PSIA XE-150 with a cantilever tip with minimal force spring constant of $k=51 \mathrm{~N} / \mathrm{m}$ and whose resonant frequency is about $320 \mathrm{kHz}$. The radius of curvature of the tip is $8 \mathrm{~nm}$ and half-width angle is $18^{\circ}$. Scanning speed was typically $0.16 \mathrm{~Hz}$. All measurements (depths, angles, diameters) were extracted from the AFM image by averaging four different profiles through the crater center.
While it is straight forward to measure the in-plane morphology of the nano-holes, it is not so easy to characterize the depth with high aspect ratio thanks to AFM measurements. So, in order to compare with the AFM measurements and to definitively characterize the exact shape of the crater, selected nano-holes have been measured by direct SEM imaging after focused ion beam (FIB) sectioning. A dual SEM/FIB device (LED 4400 (SEM)/Orsay-Physics 31 (FIB)) has been used to section the nano-holes and their imaging have been performed with a high resolution SEM (Raith-Eline). After FIB milling and a sample tilt of $\alpha\left(\alpha=45^{\circ}\right.$ or $\left.50^{\circ}\right)$ in the SEM, the projected images of the sectioned holes have been observed. Thus, the true heights $h$ are given by $h=h$ measured $/ \sin \alpha$.

\section{Results}

\subsection{Polarization dependence}

Under linear polarization ablation, it has been reported that in metals, craters exhibit a strong anisotropy [18]. However, the effect of polarization on ablation efficiency resides in the fact that light reflection on crater walls strongly depends on polarization through Fresnel coefficients, thus changing the energy deposition in the crater area [19]. We report here the case of polarization effects in single-shot ablation of borosilicate glass. Linearly polarized femtosecond laser ablation leads to strongly anisotropic nano-craters whose induced strains provoque fractures, as it can be observed by SEM examination (Fig. 1a). On the contrary, for identical fluence, circular polarization leads to quasi-perfectly symmetrical craters (Fig. 1b). Little et al [20] have recently exhibited the efficiency increase on index modification by circular polarization compared to linear one. Even at laser fluences the closest to the ablation threshold, we never retrieved the morphology of craters drilled with linearly polarized laser pulses. Birefringence effects are certainly involved, as reported by Kazansky et al. [21] who have also shown birefringence effects on laser writing on dielectrics [2,21]. Following studies have been conducted for an incident circular polarization.

\subsection{Investigation of the focal region as a function of fluence and NA}

The laser fluence $F$ has been calculated from the measured laser power $P$ and the beam waist area $\pi \omega_{0}^{2}$ (Eq. 1 ). If $d$ is the

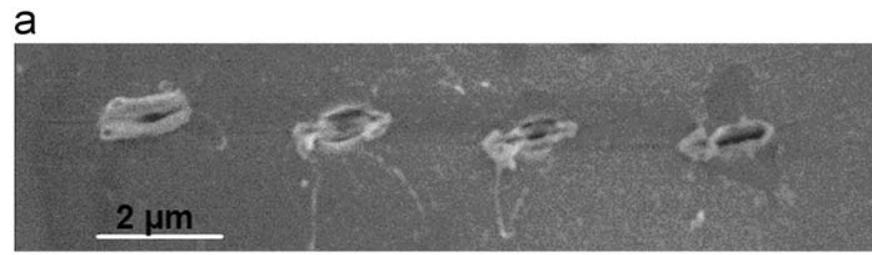

b

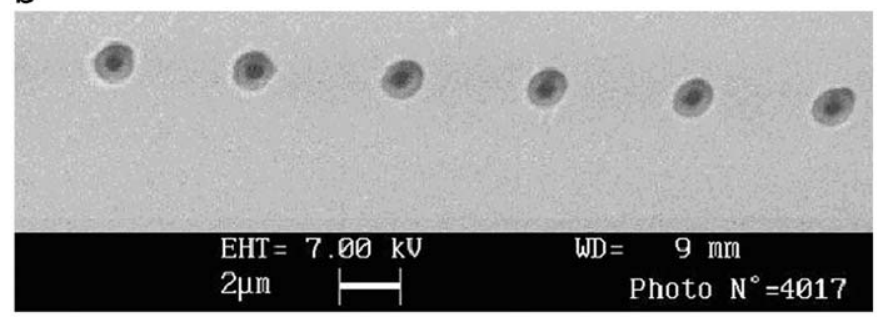

Fig. 1. SEM views of nanocraters drilled by (a) linearly polarized femtosecond pulses. The anisotropy of the crater is directed along the polarization direction. (b) Circularly polarized pulses. These two pictures have been obtained with the same fluence. 
Gaussian beam diameter measured at $1 / e^{2}$ of the peak intensity of the laser $(d \sim 15 \mathrm{~mm}$ ) and $D \sim 8 \mathrm{~mm}$ the aperture diameter of the lens, then $d / D \sim 2$ and the beam waist radius $\omega_{0}$ is given by $\omega_{0}=0417 \lambda / \mathrm{NA}$. Note that for a classical Airy disk $\omega_{0}=0.61 \lambda / \mathrm{NA}$.

$F=\frac{P}{\pi \omega_{0}^{2} f} \quad$ with $\quad \omega_{0}=\frac{0.417 \lambda}{\text { NA }}$.

In this relation, $f=5 \mathrm{kHz}, \lambda=800 \mathrm{~nm}$ and $\mathrm{NA}=0.4$ or 0.8 .

Figs. 2a,b show, as a function of the fluence for $\mathrm{NA}=0.4$ and 0.8 , the length $\Delta Z$ of the focal region where visible laser-surface sample interaction has been detected by SEM imaging. An example of such SEM imaging is shown in Fig. 3. Indeed, $\Delta Z$ $(\mu \mathrm{m})=0.25 \mathrm{~N}$, where $N$ is the number of observable impacts. Note that the reproducibility of these determinations from one specimen to another one is fairly good (Figs. 2a,b). Of course, $\Delta Z$ is an increasing function of $F$ and a decreasing function of NA, with an ablation threshold fluence $F_{0}$ of the order of $2.75 \mathrm{~J} \mathrm{~cm}^{-2}$.

Considering a Gaussian beam propagating in free space, the spot size $\omega(z)$ along the optical axis $z$ is given by

$\omega(z)=\omega_{0} \sqrt{1+\left(\frac{z}{z_{0}}\right)^{2}}$ with $z_{0}=\frac{\pi \omega_{0}^{2}}{\lambda M^{2}}$,

where $z_{0}$ is the Rayleigh range. $M \geq 1$ is a parameter which characterizes the divergence of the beam induced by the diffraction. For a perfect Gaussian beam, $M=1$. Considering the fluence threshold $F_{0}$, it is easy to show that $\Delta Z$ measured in the air is given by the following relation:

$\Delta Z=2 \gamma z_{0} \sqrt{\frac{F}{F_{0}}-1}$ with $z_{0}=\frac{\pi \omega_{0}^{2}}{\lambda M^{2}} \quad$ and $\quad \omega_{0}=\frac{0.417 \lambda}{\mathrm{NA}}$,

a

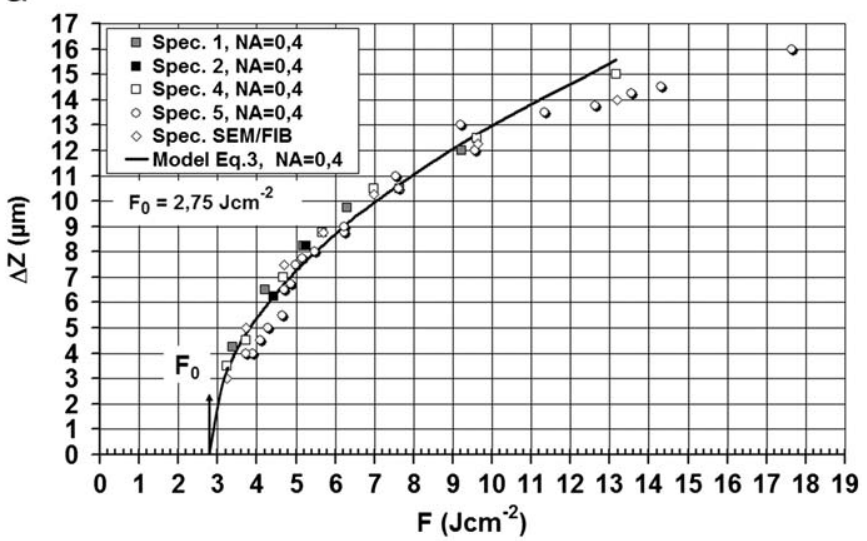

b

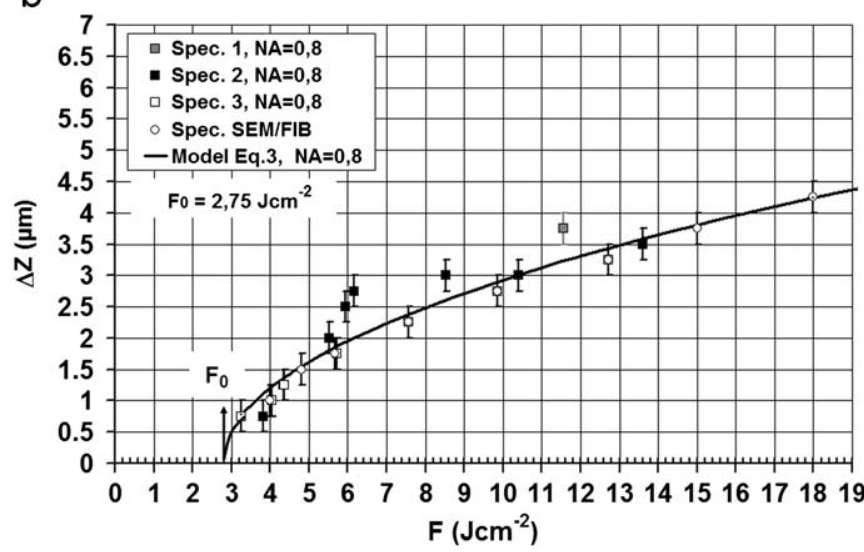

Fig. 2. Evolution of the length $\Delta Z$ of the focal region where the laser surface sample interaction is visible by direct imaging: (a) $N A=0.4$, (b) $N A=0.8$.

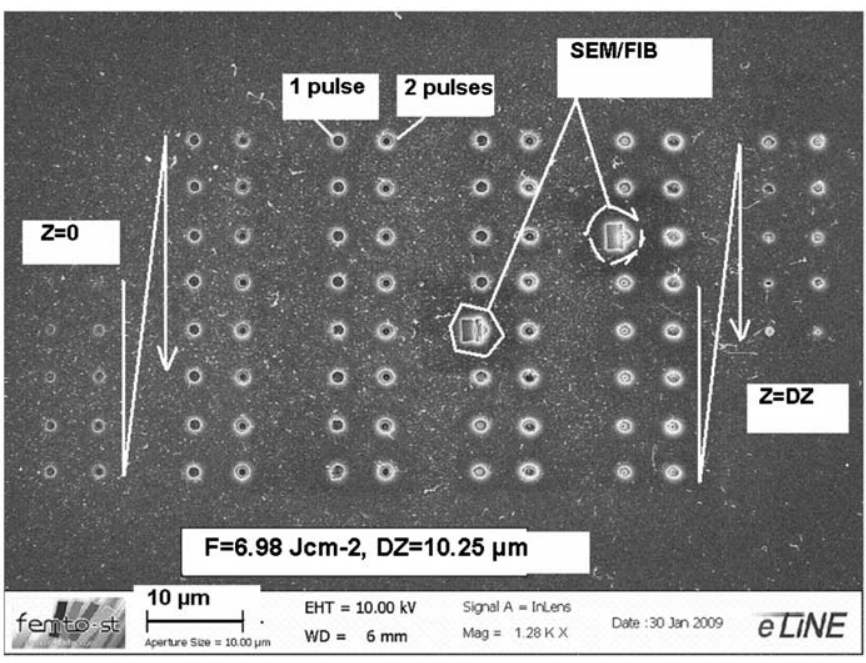

Fig. 3. Example of a global view of the laser shots path for the $\Delta Z$ determination Case of $F=6.98 \mathrm{~J} \mathrm{~cm}^{-2}$, NA=0.4, thus $\Delta Z=10.25 \mu \mathrm{m}$. In this picture we can see the tracks of the SEM/FIB machining of two holes.

where $\omega_{0}$ is the beam waist in air and $\gamma$ a fit parameter. The two unknown parameters are $F_{0}$ and $\gamma / M^{2}$. The experimental points of Figs. 2a,b have been fitted by the relation (3) (continuous curves in Figs. 2a,b) and a value of $F_{0}=2.75 \mathrm{~J} \mathrm{~cm}^{-2}$ has been determined. Moreover, $\gamma / M^{2}=1.47$ for $N A=0.4$ and $\gamma / M^{2}=1.32$ for $N A=0.8$, thus the mean value of $\gamma / M^{2}$ is of the order of 1.45 . In this last case, the uncertainties on the $\Delta Z$ measurements are not neglectible due to the low values of $\Delta Z$ (small number of impacts) and the relatively large steps $(0.25 \mu \mathrm{m})$ of the translation through the focal zone. Indeed, from Eq. (3), it is easy to show that

$\Delta Z(\mathrm{NA}=0.8)=\Delta Z(\mathrm{NA}=0.4) / 4$ if $\quad M=1$.

The $F_{0}=2.75 \mathrm{~J} \mathrm{~cm}^{-2}$ value is in accordance with previously published works on fused silica; $F_{0} \approx 2 \mathrm{~J} \mathrm{~cm}^{-2}$ for $\lambda=825 \mathrm{~nm}$, $\tau=120 \mathrm{fs}$ [22], $F_{0}=2.5 \mathrm{~J} \mathrm{~cm}^{-2}$ for $\lambda=800 \mathrm{~nm}, \tau=120 \mathrm{fs}$ [23] and $2<F<3 \mathrm{~J} \mathrm{~cm}^{-2}$ for $\lambda=798 \mathrm{~nm}, \tau=120 \mathrm{fs}$ [24]. Note that the value of the threshold depends on the wavelength $\lambda$ and on the pulse duration $\tau$ [22-24].

\subsection{General morphology of the nano-holes}

As a function of the fluence and the NA, two kinds of morphologies have been observed composed of only one crater or of two characteristic craters (appearance of a secondary crater) when laser self-focusing appears [25]. The conditions of the selffocusing appearance will be described later. Figs. 4a,b show the typical morphology, without self-focusing, of a nano-crater generated by single-shot ablation of silica $\left(F=5.69 \mathrm{~J} \mathrm{~cm}^{-2}\right.$, $N A=0.4$ ) imaged by AFM (a) and SEM (b) after MEB/FIB crosssectioning. Figs. 5a,b show the same pictures with the two characteristic craters when self-focusing occurs $(F=5.87$ and $7.56 \mathrm{~J} \mathrm{~cm}^{-2}$ with $\mathrm{NA}=0.8$ ). The craters are quasi-axisymmetric along the laser beam axis and surrounded by a hemi-torus rim. The self-focusing crater is conical with a big aspect ratio, whereas the profile of the principal crater is trapezoidal or more exactly looks like a diabolo. This morphology is typical of thermal ablation. However, typical morphology of Coulomb explosion, i.e. soft ablation with clean craters and no protrusion [10], has never been observed in this study even for craters depths down to some tens of nanometers. Petrov and Davis [13] have recently shown that during ultrafast ablation of dielectrics, collisional ionization is the dominant ionization mechanism, which is due to 


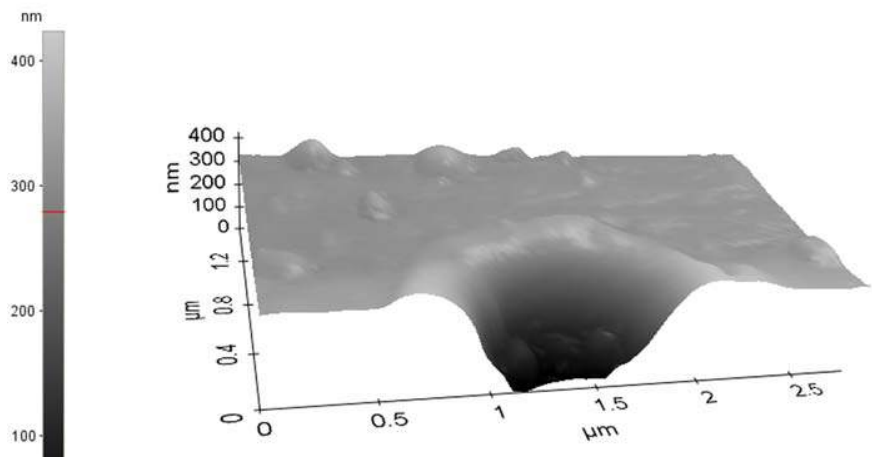

100

b

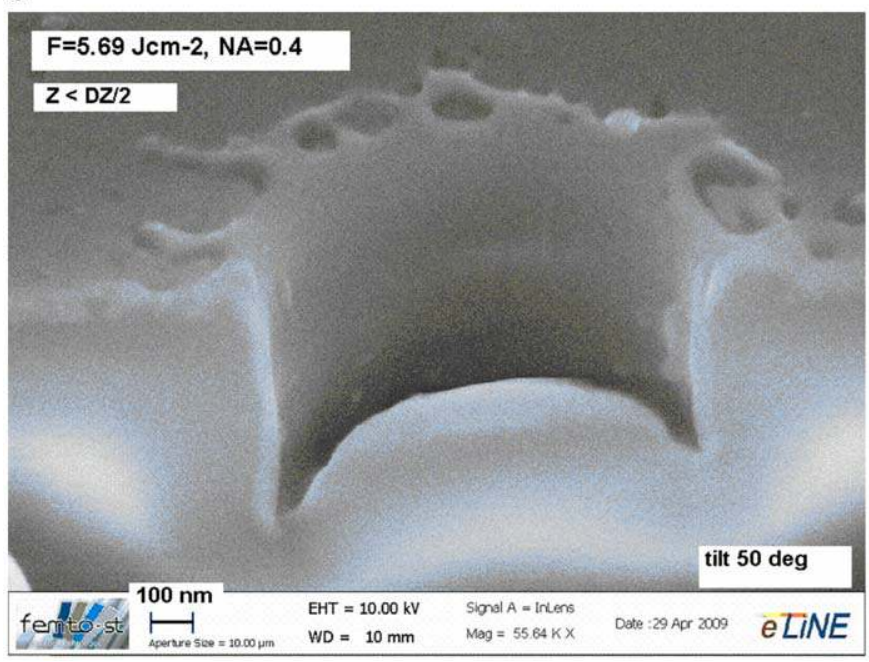

Fig. 4. (a) AFM and (b) SEM imaging of the same crater for $F=5.69 \mathrm{~J} \mathrm{~cm}^{-2}$, NA=0.4 without self-focusing.

the heating of conduction band electrons and therefore follows linear absorption.

Comparing the AFM and the SEM pictures, due to the opening angle of the AFM tip $\left(\Delta \theta / 2=18^{\circ}\right)$, it is clear that the AFM profiles are not exact. The inclination of the sides and the diameter of the bottom of the crater are underestimated, and moreover the height of the secondary crater is not measurable. These two points will be discussed later. From a qualitative point of view, Fig. 6 gives the scheme of a typical profile and the different characteristic dimensions (heights: $h_{1}, h_{2}, h_{3}$ and diameters: $L_{1}, L_{2}, L_{3}, L_{4}, L_{5}$ ) which have been systematically investigated in AFM and SEM pictures.

3.4. Evolution through the focal region of the characteristic dimensions of the craters

\subsubsection{Study of principal crater: $h_{1}, L_{1}$ and $L_{2}$ parameters}

Thanks to AFM imaging the different dimensions of the craters have been evaluated through the focal zone, along the $Z$ direction. The origin $z=0$ corresponds to the first observable impact (see Fig. 3) when the waist of the beam is outside of the glass and $z=\Delta Z$, to the last visible impact (Fig. 3 ) with the focal point inside the glass. To obtain a symmetric abscissa with respect to the origin, whatever the fluence, thus $\Delta Z$, the evolutions of the parameters are plotted as a function of $z-\Delta Z / 2(z=-\Delta Z / 2$ for $z=0$
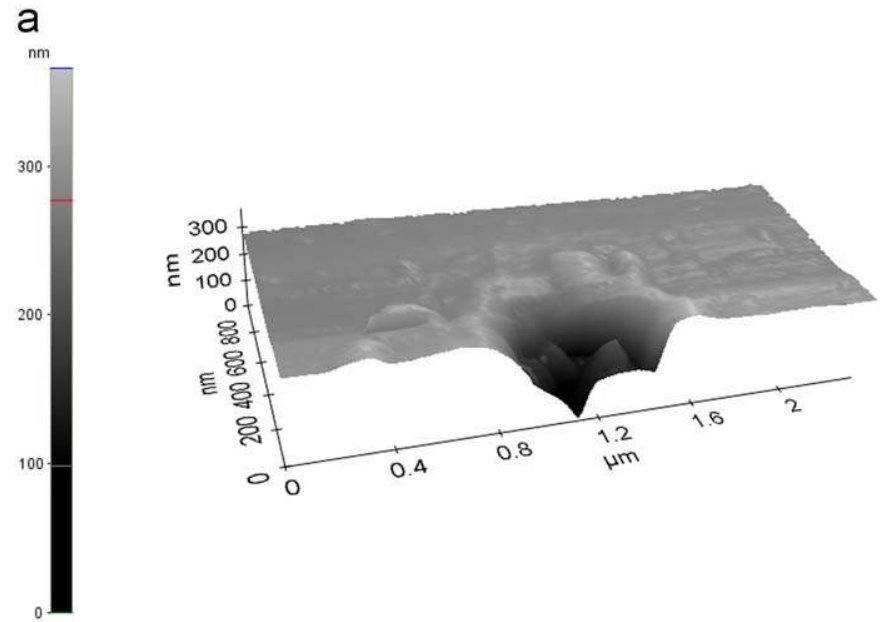

b

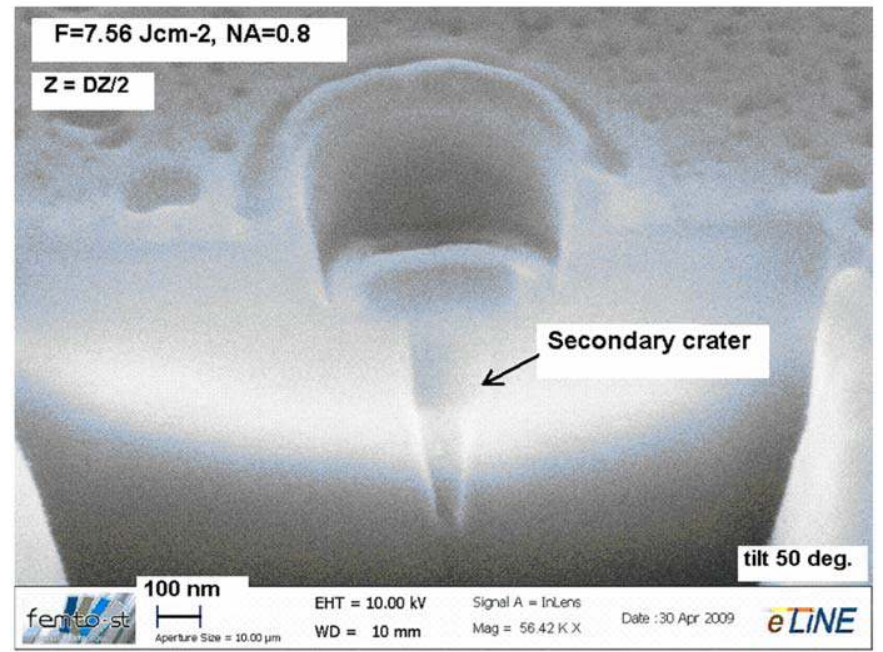

Fig. 5. (a) AFM $\left(F=5.87 \mathrm{~J} \mathrm{~cm}^{-2}\right)$ and (b) $\operatorname{SEM}\left(F=7.56 \mathrm{~J} \mathrm{~cm}^{-2}\right)$ imaging of craters for $\mathrm{NA}=0.8$ with self-focusing craters.

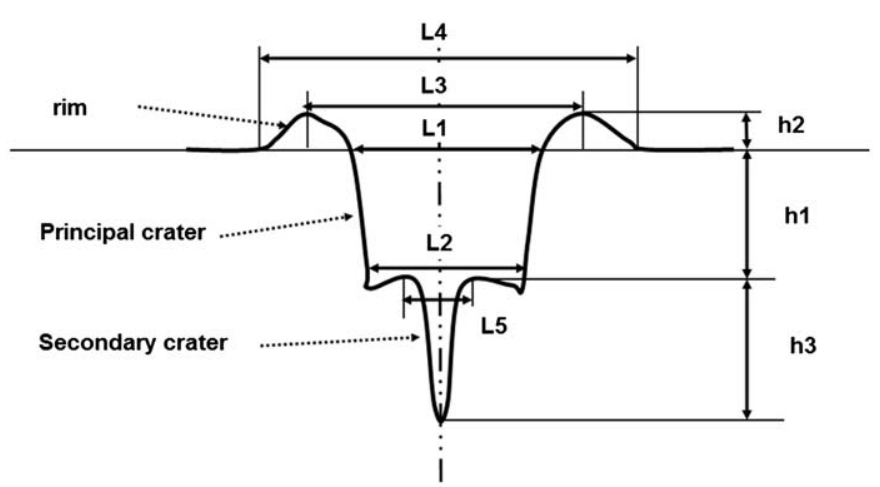

Fig. 6. Diagram of the crater morphology. Definition of the different dimensions.

and $z=\Delta Z / 2$ for $z=\Delta Z$ ). Fig. 7a shows, for different fluences (specimen 4 with $N A=0.4$ ), the evolution of the heights $h_{1}$ of the craters as a function of $z-\Delta Z / 2$. All of the profiles in Fig. 7a are nearly symmetrical with respect to the $X$ axis and maximum for $z \sim \Delta Z / 2$, when the fluence is maximum, that is to say when the beam is focused at the sample surface. To normalize the representations in the Fig. $7 \mathrm{a}$ with respect to $\Delta Z$ and $h_{1}$, the new coordinates in Fig. 7b are: $z^{*}=(2 z \mid \Delta Z)-1$, thus $-1 \leq z^{*} \leq 1$, and 
a

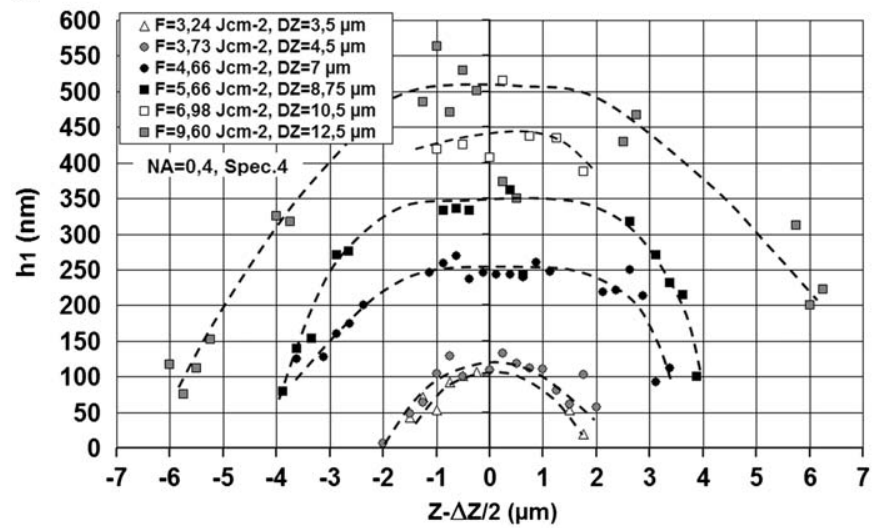

b

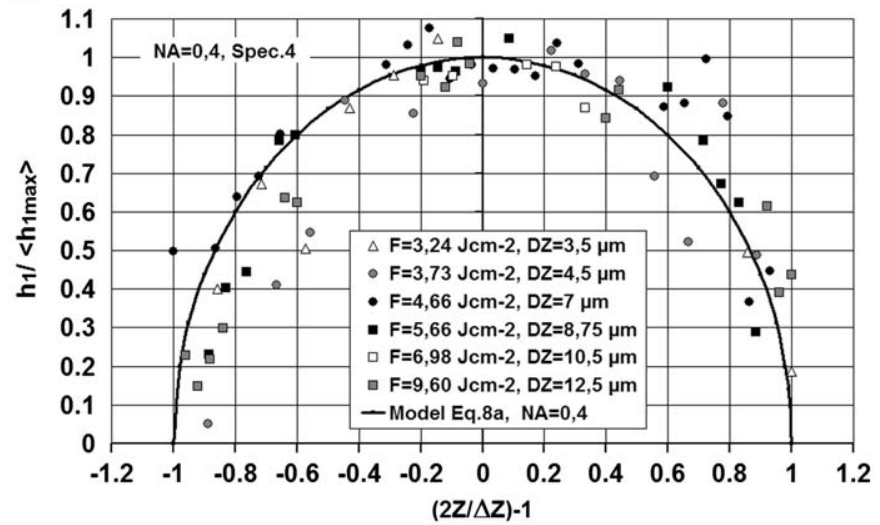

Fig. 7. Evolution for different fluences and $N A=0.4$ of (a) the depth $h_{1}$ as a function of $(z-\Delta Z / 2)$, (b) the normalized depth $h_{1} /<h_{1 \max }>$ as a function of the normalized focal position $((2 z \mid \Delta Z)-1)$. Representation of the locus given by Eq. (8a).

$h_{1} /\left\langle h_{1 \max }\right\rangle$, where $\left\langle h_{1 \max }\right\rangle$ is the maximum mean value of the height $h_{1}$ for each studied fluence. So, whatever the fluence is, and then $\Delta Z$, all points are appreciably on the same locus. The same procedure has been applied with $\mathrm{NA}=0.8$, all the points ( $\mathrm{NA}=0.4$ and $N A=0.8$ ) belong to the same locus. Figs. 8a,b show the evolutions of the diameters $L_{1}$ of the same craters as those of Figs. 7a,b and in the same system of coordinates. As for the depths $h_{1}$, the diameters measures belong to the same master curve, whatever the NA and the fluence are.

In the case of low fluences, the laser energy is mainly deposited in the shallow region defined by the optical penetration depth. Due to non-linear nature of the interaction of the femtosecond laser pulses with transparent material, simultaneous absorption of several photons is required to initiate ablation. Multi-photon absorption produces free electrons which are accelerated by the electric field of the laser. These electrons induce avalanche ionization and optical breakdown and thus generate a microplasma. The expansion of this plasma creates nano-structure on the surface of the sample. Each discrete photon of the laser beam is described by a Gaussian distribution in both time and space. So, if each photon is absorbed according to a look like Beer-Lambert law for Gaussian pulses, thus

$I\left(x, z^{\prime}\right)=I_{0} e^{-A(\alpha, q, R) z^{\prime}} e^{-\left(x / x_{0}\right)^{2}}$

where $z^{\prime}$ is the depth measured from the surface of specimen, $x$ the lateral distance from the optical axis, $x_{0}$ the width of the pulse and $A(\alpha, q, R)$ a absorption coefficient which depends on the linear a

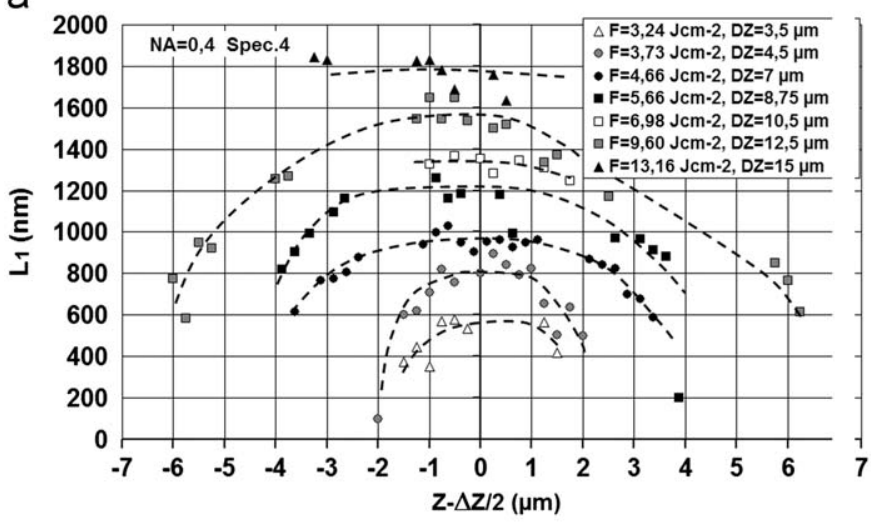

b

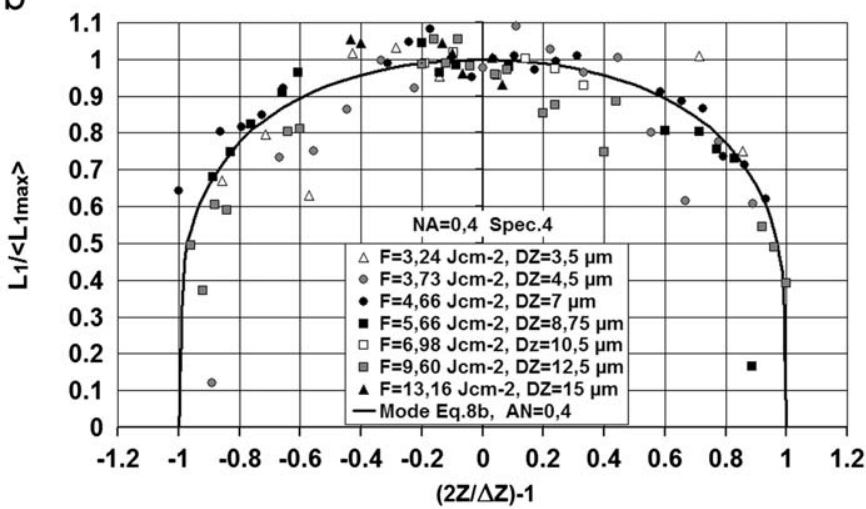

Fig. 8. Evolution for different fluences and $N A=0.4$ of (a) the diameter $L_{1}$ as a function of $(z-\Delta Z / 2)$, (b) the normalized diameter $L_{1} /<L_{1 \max }>$ as a function of the normalized focal position $((2 z \mid \Delta Z)-1)$. Representation of the locus given by Eq. (8b).

absorption coefficient $\alpha$ of the material, the number $q$ of required photons to initiate ablation ( $q=6$ for $\mathrm{SiO}_{2}$ ) and the reflectivity $R$ of the surface [26]. Hence, from Eq. (5) and as shown in the literature $[8,16,22,26]$, a logarithmic dependence of the depth $h_{1}$ and the width $L_{1}$ of the craters as a function of the fluence $F$ is deduced as follows:

$h_{1}=h_{10} \operatorname{Ln} \frac{F}{F_{0}} \quad$ and $\quad L_{1}=L_{10}\left(\operatorname{Ln} \frac{F}{F_{0}}\right)^{1 / 2}$,

where $h_{10}$ and $L_{10}$ are two dimensional constants which are dependent on the ablated material and the beam characteristics, respectively.

Thus, from Eq. (6a,b):

$\frac{h_{1}}{h_{10}}=\left(\frac{L_{1}}{L_{10}}\right)^{2}$

The height of the crater varies as the square of his diameter. The relation (7) is fairly well verified on the whole of the measured craters, whatever $z$, and in particular, as it will be shown later, on the maximum mean values $\left\langle h_{1 \max }\right\rangle$ and $\left\langle L_{1 \max }\right\rangle$. Taking into account the relations (2) (5) and (7) the locus of the points $h_{1} /\left\langle h_{1 \text { max }}\right\rangle$ (Fig. 7b) and of $L_{1} /\left\langle L_{1 \text { max }}\right\rangle$ (Fig. 8b) can be written as

$\frac{h_{1}}{\left\langle h_{1 \max }\right\rangle}=\left(1-\left(\frac{2 z}{\Delta Z}-1\right)^{2}\right)^{1 / 2}$ and $\frac{L_{1}}{\left\langle L_{1 \max }\right\rangle}=\left(1-\left(\frac{2 z}{\Delta Z}-1\right)^{2}\right)^{1 / 4}$.

These two relations have been drawn in the Figs. 7(b) and 8(b) and are in a fairly good agreement with the experimental points. 


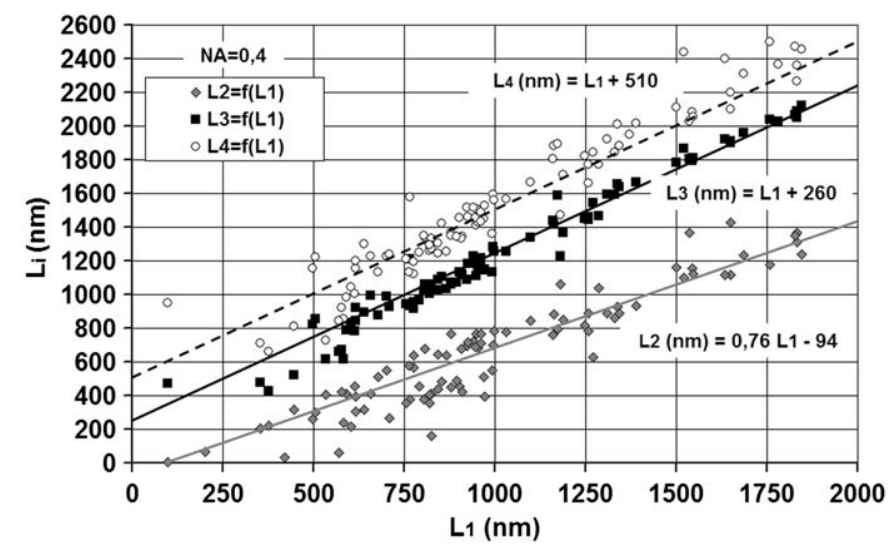

Fig. 9. Evolution of the diameters $L_{2}, L_{3}$ and $L_{4}$ as a function of the upper diameter $L_{1}$ for (a) $N A=0.4$

Now, considering the diameter $L_{2}$ of the bottom of the craters and considering the half opening angle of the AFM tip, $\Delta \theta / 2$, the true value of $L_{2}$ has been deduced from the measured one $L_{2 \mathrm{mes}}$, by

$L_{2}=L_{2 \mathrm{mes}}+2 h_{1} \operatorname{tg}(\Delta \theta / 2)$.

Note that to determine in a more accurate manner the values of $L_{2 \max }$ (idem for $L_{4}$ and $L_{5}$ ), a Sobel filter has been applied on the AFM images, which gives the derivative image of the profile. Hence, the fast changes in the slopes of the profiles are enhanced. As it is shown in Fig. 9, where the $L_{2}$ values (and also $L_{3}$ and $L_{4}$ ) have been plotted as a function of the $L_{1}$ values for NA=0.4, and as it will be shown later on the maximum value of $L_{2}$, whatever the fluence and the NA are, a linear relationship has been found between these two diameters:

$L_{2}=\alpha_{21} L_{1}-\beta_{21}$ with $\alpha_{21}=0.76$.

$\beta_{21} \sim 100 \mathrm{~nm}$ for $\mathrm{NA}=0.4$ and $\beta_{21} \sim 50 \mathrm{~nm}$ for $\mathrm{NA}=0.8$.

Hence, the relations (10) and (7) involve a decrease of the walls inclination as $1 / L_{1}$, thus with the fluence increasing. For very small impacts, $L_{2}=0$ and $L_{1}=\beta_{21} / \alpha_{21}$, the shape is nearly conical with a large opening angle $\left(>50^{\circ}\right)$.

Figs. 10a,b, 11 and 12 give, for the different specimens, the evolutions of the maximum mean values of the depths $\left\langle h_{1 \max }\right\rangle$ and of the diameters $\left\langle L_{1 \max }\right\rangle,\left\langle L_{2 \max }\right\rangle$, respectively, as a function of fluence and NA. As previously shown, these maxima are measured in the focal region in the neighborhood of $z \sim \Delta Z / 2$. Note that the AFM and the SEM/FIB determinations are in a fairly good agreement.

Following the absorption law given by Eq. (5) and according to the relations (6a,b) and (10), the variations of $\left\langle h_{1 \max }\right\rangle,\left\langle L_{1 \max }\right\rangle$ and $\left\langle L_{2 \max }\right\rangle$ are written as

$$
\begin{aligned}
& \left\langle h_{1 \max }\right\rangle=h_{10} \operatorname{Ln}\left(\frac{F}{F_{0}}\right) \\
& \left\langle L_{1 \max }\right\rangle=L_{10}\left(\operatorname{Ln}\left(\frac{F}{F_{0}}\right)\right)^{1 / 2} \\
& \left\langle L_{2 \max }\right\rangle \cong 0.76\left\langle L_{1 \max }\right\rangle
\end{aligned}
$$

These relations are plotted in Figs. 10a,b, 11 and 12 with $h_{10}=490 \mathrm{~nm}$ and $245 \mathrm{~nm}$ and $L_{10}=1360 \mathrm{~nm}$ and $670 \mathrm{~nm}$, for $\mathrm{NA}=0.4$ and 0.8 , respectively. They fairly match all experimental data. Moreover, a factor two on the $h_{10}$ and $L_{10}$ values with respect to the NA has been pointed out, thus, we can deduce that

$\mathrm{NA} h_{10}=c^{t e}=200 \mathrm{~nm}$

$\mathrm{NA} L_{10}=c^{t e}=540 \mathrm{~nm}$ a

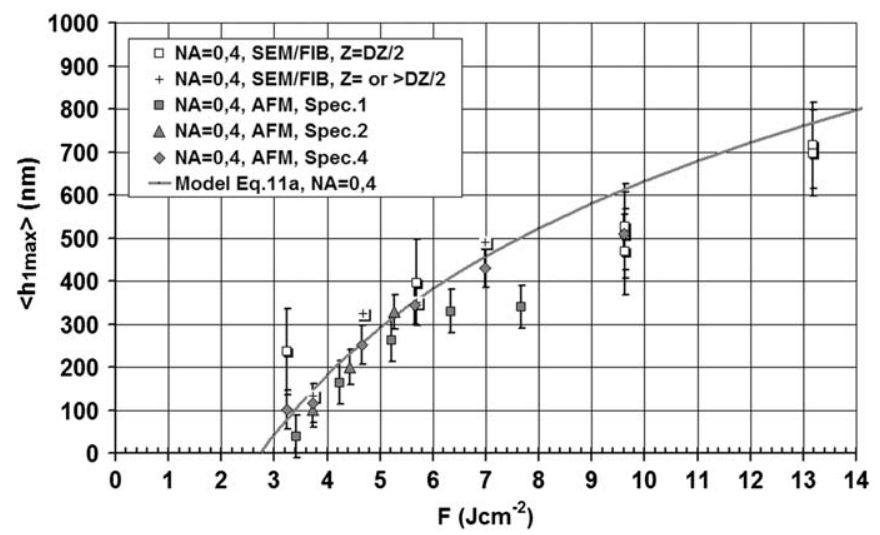

b

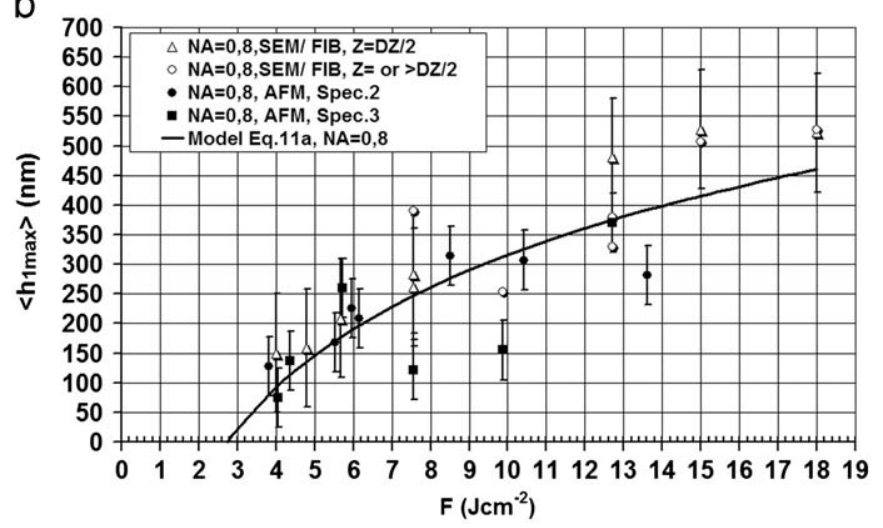

Fig. 10. Variation of the maximum depth $<h_{1 \max }>$ as a function of the fluence $F$ for (a) $N A=0.4$ and (b) $N A=0.8$. Comparison of the measures obtained by AFM and SEM/FIB imaging. Representation of Eq. (11a).

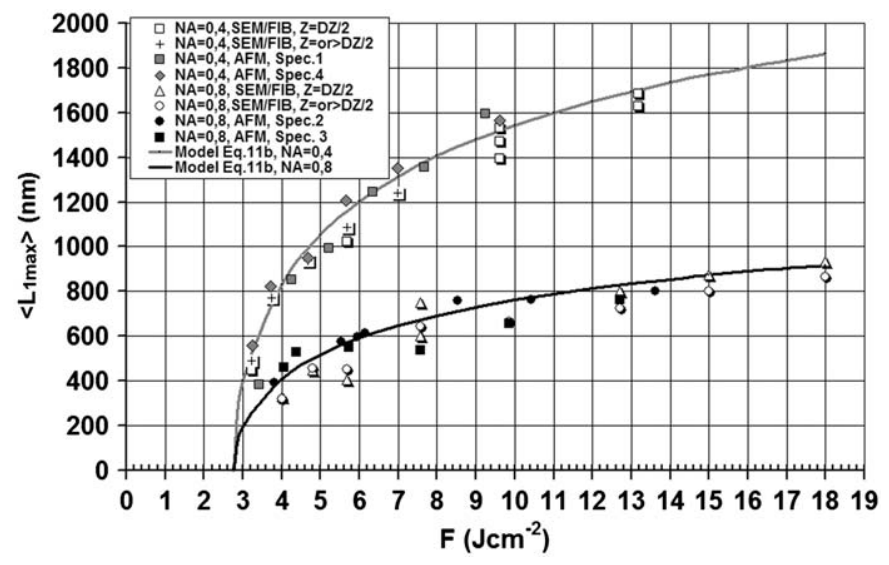

Fig. 11. Variation of the maximum of the upper diameter $<L_{1 \max }>$ as a function of the fluence for $\mathrm{NA}=0.4$ and 0.8 . Comparison of the measures obtained by AFM and SEM/FIB imaging. Representation of Eq. (11b).

The aspect ratio of the principal craters is small, $h_{10} / L_{10} \sim 0.37$. It is interesting to note that $L_{10} / 2$ values correspond to about $81 \%$ of the beam waist $\omega_{0}$ Eq. (3) for the two NA, which agrees with Eq. (5) in which $x_{0}=2 \omega_{0}$ and those reported by Koch et al. [8] where $L_{10}=2 \omega_{0}$. For linear absorption, $h_{10}$ is close to the inverse of the absorption coefficient $\alpha$ of the material, $\alpha^{-1} \sim 100 \mu \mathrm{m}$ for silica, thus a few hundred times larger than the value given in Eq. (12). The $h_{10}$ value is linked with the absorption coefficient 


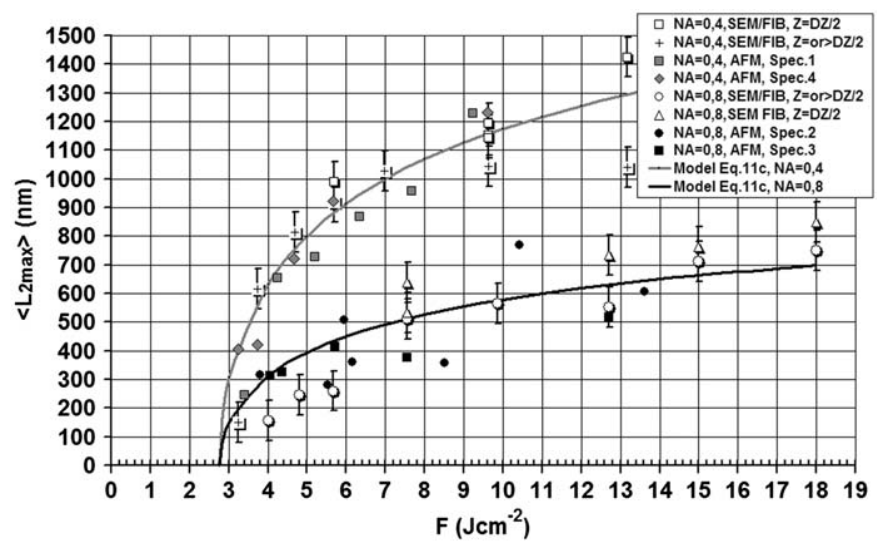

Fig. 12. Evolution of the maximum of the lower diameter $\left\langle L_{2 \max }>\right.$ as a function of the fluence for $\mathrm{NA}=0.4$ and 0.8 . Comparison of the measures obtained by AFM after tip correction (Eq. (9)) and SEM/FIB imaging. Representation of Eq. (11c).

$A(\alpha, q, R)$ in Eq. (5). According to Hwang et al. [26], for non-linear absorption, instead the optical penetration depth, $h_{10}$ stands for the effective energy penetration depth.

\subsubsection{Study of the rim: $h_{2}, L_{3}$ and $L_{4}$ parameters}

A rim protrusion is visible around all craters (Figs. 4 and 5). First, during phase explosion, numerous small clusters are ejected and condensated, especially when the ablation is performed under atmospheric pressure. Second, thermocapillary forces can extract liquid material out of the center of the crater, due to thermal gradients. This effect is known as Marangoni effect [17]. From Fig. 9, for NA=0.4 (Spec. 4), where $L_{3}$ and $L_{4}$ have been plotted as a function of the diameter $L_{1}$ of the craters, a linear relationship can be drawn, as previously mentioned for $L_{2}$. Thus,

$L_{3}=L_{1}+\beta_{31}$

$L_{4}=L_{1}+\beta_{41}$

In a first approximation, for the two NA, the width of the rim $\beta_{41} / 2$ is constant and equal to about $300 \mathrm{~nm}$. Moreover, as $\beta_{31} \sim \beta_{41} / 2$, the shape of the rim can be approximated by a hemi-torus whose cross-section is half elliptical (Figs. 4 and 5). The two principal axis of the ellipse are $\beta_{41} / 2$ and $h_{2}$. Note that, when there is no observable crater $\left(L_{1}=0\right), L_{3}$ and $L_{4}$ values are positive. This situation has been especially observed at the end of the focal excursion $(z \sim \Delta Z)$ and for only one to three impacts. In this case, the height of these bumps is great compared to those measured when well-defined craters exist. It is possible that the craters are plugged by the material and so not observable. The evolution of $h_{2}$ as a function of $z-\Delta Z / 2$ follows the same tendency as those reported on Figs. 7a and 8a for $h_{1}$ and $L_{1}$. Moreover, although there is a great scattering on the $h_{2}$ values, $h_{2}$ globally increases with the fluence and seems to be independent of the NA for a given fluence; $h_{2}$ increases from $15 \pm 10 \mathrm{~nm}$ for $F=3.2 \mathrm{~J} \mathrm{~cm}^{-2}$ to $60 \pm 7 \mathrm{~nm}$ for $F \sim 10 \mathrm{~J} \mathrm{~cm}^{-2}$. Thus, knowing $h_{1}, L_{1}, L_{2}$ and $h_{2}, L_{4}$, the volume $V_{h}$ of the holes (truncated cone) and the volume of the corresponding rim $V_{r}$ have been calculated according to

$V_{h}=\frac{\pi h_{1}}{12}\left(L_{1}^{2}+L_{1} L_{2}+L_{2}^{2}\right) \quad$ and $\quad V_{r}=\frac{\pi^{2}}{16} h_{2}\left(L_{4}^{2}-L_{1}^{2}\right)$.

The relation between $V_{r}$ and $V_{h}$ has been drawn in Fig. 13. In a ratio equals to two with respect to the mean value of the rim volume, it is shown that for the smallest holes $\left(V_{h} \leq 10^{-2} \mu \mathrm{m}^{3}\right)$, the volume of the rim seems to be of the order of those of the craters, but for the bigger holes, $V_{r}<V_{h}$. In fact, when the fluence increases, small clusters are ejected and condensed far away of the

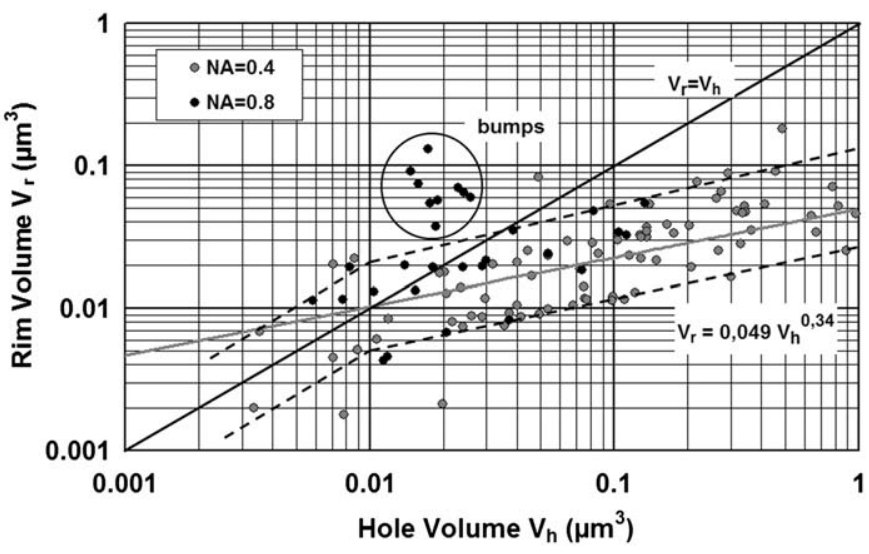

Fig. 13. Volume $V_{r}$ of the rim as a function of the volume $V_{h}$ of the principal crater.

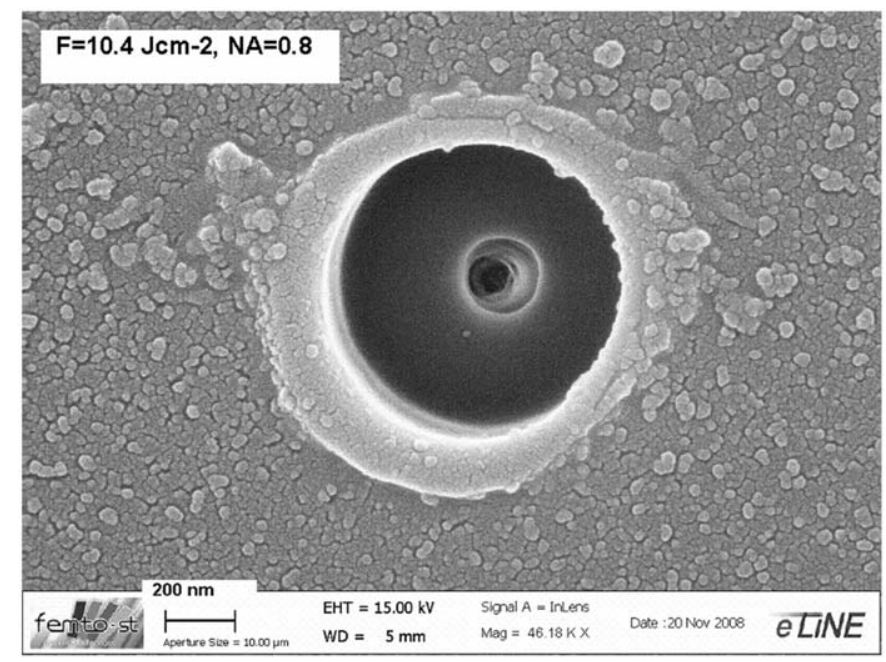

Fig. 14. SEM image of the principal and the self focusing craters. $F=10.4 \mathrm{~J} \mathrm{~cm}^{-2}$ and $\mathrm{NA}=0.8$.

craters and do not participate to the rim formation, thus $V_{r}<V_{h}$. In this case, a fit of the experimental points gives

$V_{r}\left(\mu \mathrm{m}^{3}\right) \sim 5.10^{-2} V_{h}^{0.34}$.

\subsubsection{Study of the secondary craters: $h_{3}$ and $L_{5}$ parameters}

The thin, long conical profile shown in Figs. 5a,b suggests that there is self-focusing of the laser pulse as it propagates into the glass with a sub-diffraction sized diameter. This is due to the Kerr effect; the non-zero non-linear refractive index $n_{2}$ gives many non-linear optical effects such as intense laser pulse propagation through the dielectric material. Indeed, the spatial variation of the refractive index causes a lens-like effect that tends to focus the laser beam inside the material (Fig. 14). However, others mechanisms as the micro-plasma formation can defocus the beam and possibly balancing the self-focusing leading to self-channeling of the pulse inside the dielectric. Note that self-focusing is predicted if the power peak of the laser pulse exceeds a critical power $P_{C}$ given by $[9,27]$

$P_{C}=\frac{(0.61)^{2} \pi \lambda^{2}}{8 n_{0} n_{2}}$.

$n_{0}$ and $n_{2}$ are the linear and non-linear part of the refractive index of the material. With $n_{0}=1.46$ and $n_{2} \sim 3.7 \times 10^{-16} \mathrm{~cm}^{2} \mathrm{~W}^{-1}$ 
for the silica, thus $P_{C} \sim 1.7 \mathrm{MW}$ corresponding to a pulse energy $E_{C}$ of the order of $0.21 \mu \mathrm{J}$.

Thanks to AFM and SEM/FIB imaging, the appearance of self-focusing craters has been observed through the focal region in the neighborhood of $z \sim \Delta Z / 2$; i.e. when the beam is focused inside the material. More exactly, self-focusing occurs when $z \geq \Delta Z / 2+1.7 \pm 0.25 \mu \mathrm{m}$ for $\mathrm{NA}=0.4$ and $z \geq \Delta Z / 2-0.45 \pm 0.25 \mu \mathrm{m}$ for $N A=0.8$. This observation agrees with the hypothesis of a displacement of the waist of the beam in the opposite direction of the beam propagation. Moreover, self-focusing has not been observed for very low fluences, near the threshold $F_{0}$; i.e. below $F_{C}$ $\sim 4 \pm 0.5 \mathrm{~J} \mathrm{~cm}^{-2}$ and whatever the NA is. This fluence value corresponds to a pulse energy $E_{C}$ of $\sim 0.09 \pm 0.011 \mu \mathrm{J}$ for $\mathrm{NA}=0.4$ and $\sim 0.02 \pm 0.01 \mu \mathrm{J}$ for $\mathrm{NA}=0.8$, thus, lower than those predicted by Eq. (16), $E_{\mathrm{c}} \sim 0.21 \mu \mathrm{J}$. However, taking into account the laser beam intensity at $1 / e^{2}$ with $d / D \sim 2$ (as in Eq. (1)), the numerical factor in Eq. (16) is equal to 0.41 and the new value of the critical energy $E_{C}$ is $0.098 \mu \mathrm{J}$, thus relatively close to the experimental ones.

Except some craters (one or two) at the beginning and the end of the self-focusing appearance where the craters are very small, the diameter $L_{5}$ of the self-focusing craters is nearly constant as shown in Fig. 15, $L_{5} \sim 200 \pm 25 \mathrm{~nm}$, regardless the fluence and the NA. Note that the AFM and SEM measurements are in a fairly good agreement. The depths $h_{3}$ of the self-focusing craters have been determined from the SEM imaging of the SEM/FIB sectioned holes. Only some measurements have been carried out whose results are presented in Fig. 16. For NA=0.8, $h_{3}$ increases from $200 \pm 100$ to $1020 \pm 150 \mathrm{~nm}$ with the fluence increasing from $F=4$ to $18 \mathrm{~J} \mathrm{~cm}^{-2}$, respectively. The value determined for $\mathrm{NA}=0.4$ is greater than those obtained for $\mathrm{NA}=0.8$, but with a great uncertainty.

As soon as the strong non-linear propagation regime is concerned, the local intensity does not follow a simple relationship with the incident power and, the intensity can increase by several orders of magnitude due to spatial and temporal compression during the propagation. In the present case, taking into account the values of $L_{5}$ and of the critical fluence for the secondary crater appearance, $F_{C}=3.8 \mathrm{~J} \mathrm{~cm}^{-2}$, a peak intensity of the order of $2.3 \times 10^{15} \mathrm{~W} \mathrm{~cm}^{-2}$ for NA=0.4 has been calculated. At such intensities the deposition of the laser energy occurs at near constant volume, which is the case in this study; $V \sim 10^{-2} \mu \mathrm{m}^{3}$ with a big aspect ratio $h_{3} / L_{5}$ of the order of 4 to 5 when $F$ $>8 \mathrm{~J} \mathrm{~cm}^{-2}$, and produces a high temperature over-dense plasma causing a local micro-explosion. Note that the self-focusing craters are very clean and any rim has been observed in the vicinity of these craters (see for instance Fig. 14).

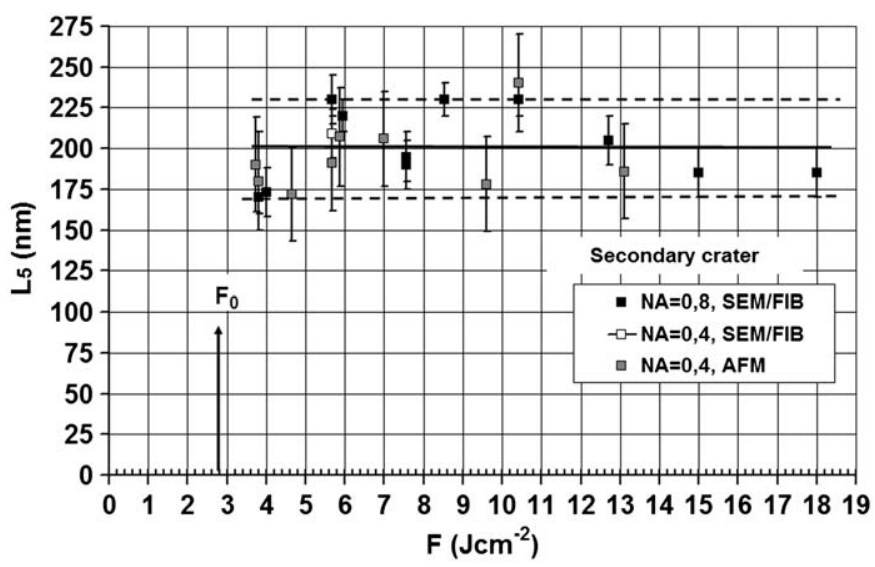

Fig. 15. Variation of the diameter $L_{5}$ of the self-focusing craters as a function of the fluence $F$ for the two NA.

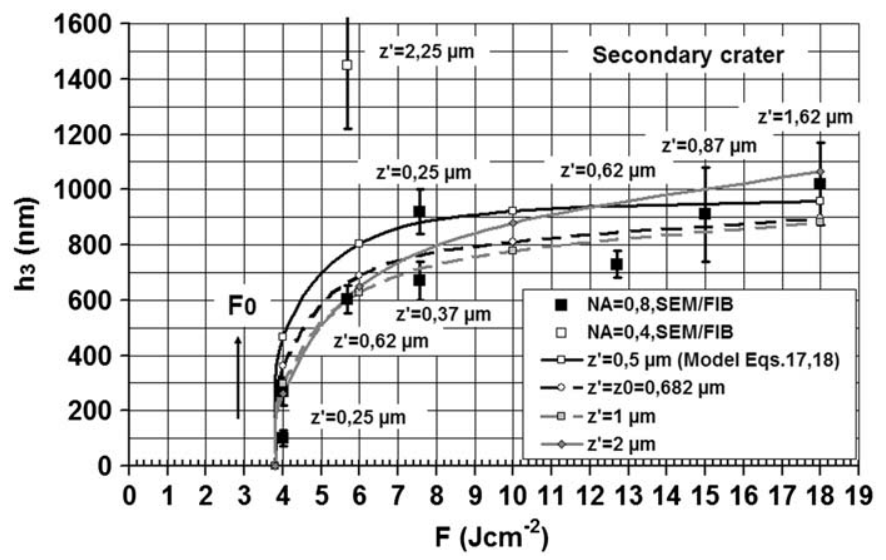

Fig. 16. Evolution of the depth $h_{3}$ of the self-focusing craters as a function of the fluence $F$ for $\mathrm{NA}=0.8$. Plot of Eqs. (17) and (18) with $\delta=0.56$ for different values of $z^{\prime}$.

However, for a Gaussian beam, self-focusing leads to a displacement $\Delta f_{S F}$ of the focal point in the opposite direction of the beam propagation. Assuming, as a very simple model, that the variations of the depth $h_{3}$ are proportional to those of the displacement $\Delta f_{S F}$, it is possible to write

$h_{3}\left(z^{\prime}, F\right)=\delta \Delta f_{S F}\left(z^{\prime}, F\right)$,

where $\delta$ is a proportionality factor (parameter of the model) and $z^{\prime}$ the propagation distance of the beam from the surface of the sample ( $z^{\prime}=0$ when $z \sim \Delta Z / 2$ ). Following Sudrie et al. [27], $\Delta f_{S F}\left(z^{\prime}, F\right)$ is given by the relation (18)

$\Delta f_{S F}=\frac{R^{2}}{R+\Delta Z_{S F}} \quad$ with $\quad R=n_{0} z^{\prime}\left(1+\left(\frac{z_{0}}{Z^{\prime}}\right)^{2}\right)$

and $\Delta Z_{S F}=\frac{z_{0}\left(1+\left(\frac{z^{\prime}}{z_{0}}\right)^{2}\right)}{n_{0} \sqrt{\frac{F}{F_{c}}-1}}$.

In this equation, $R$ is the beam curvature radius in the silica, $\Delta Z_{S F}$ the focal distance when self-focusing occurs and $z_{0}$ the Rayleigh range in air (Eq. (2) with $M=1$ ). In Fig. 16, the relation (17) with $\delta=0.56$ and $F_{C}=3.8 \mathrm{~J} \mathrm{~cm}^{-2}$ (model's parameters) is plotted as a function of the fluence for different values of $z^{\prime}$ in the range of the experimental ones where $h_{3}$ has been measured. Those different values are reported in Fig. 16. It is thus shown that there is a fairly good agreement with the experimental determinations by taking $\delta=0.56$ in this simple model.

Thus, as a conclusion of this morphology study, combining the Eqs. (3),(8),(10),(11) and (12), the variations of $h_{1}, L_{1}$ and $L_{2}$ in the focal region can be written as a function of $\Delta Z, F$, NA and the characteristics of the laser beam

$h_{1}(\mu \mathrm{m})=\frac{0.2}{\mathrm{NA}} \operatorname{Ln}\left(1+\left(\frac{\Delta Z}{2 \gamma z_{0}}\right)^{2}\right)\left(1-\left(\frac{2 z}{\Delta Z}-1\right)^{2}\right)^{1 / 2}$

$\left.L_{1}(\mu \mathrm{m})=\frac{0.54}{\mathrm{NA}} \operatorname{Ln}\left(1+\left(\frac{\Delta Z}{2 \gamma z_{0}}\right)^{2}\right)\right)^{1 / 2}\left(1-\left(\frac{2 z}{\Delta Z}-1\right)^{2}\right)^{1 / 4}$

$h_{2} \cong 0.76 \mathrm{~L}_{1} \quad$ with

$\Delta Z=2 \gamma z_{0} \sqrt{\frac{F}{F_{0}}-1}$ and $z_{0}=\frac{\pi \omega_{0}^{2}}{\lambda M^{2}}, \omega_{0}=\frac{0.417 \lambda}{N A}$,

$\gamma / M^{2} \cong 1.45, F_{0}=2.75 \mathrm{Jcm}^{-2}$

Concerning the self-focusing craters, they have been observed only for $F \geq 4 \mathrm{~J} \mathrm{~cm}^{-2}$ and $z \geq \Delta Z / 2$. Their diameter is nearly constant, $L_{5} \sim 200 \pm 25 \mathrm{~nm}$ and their depth increases with the 


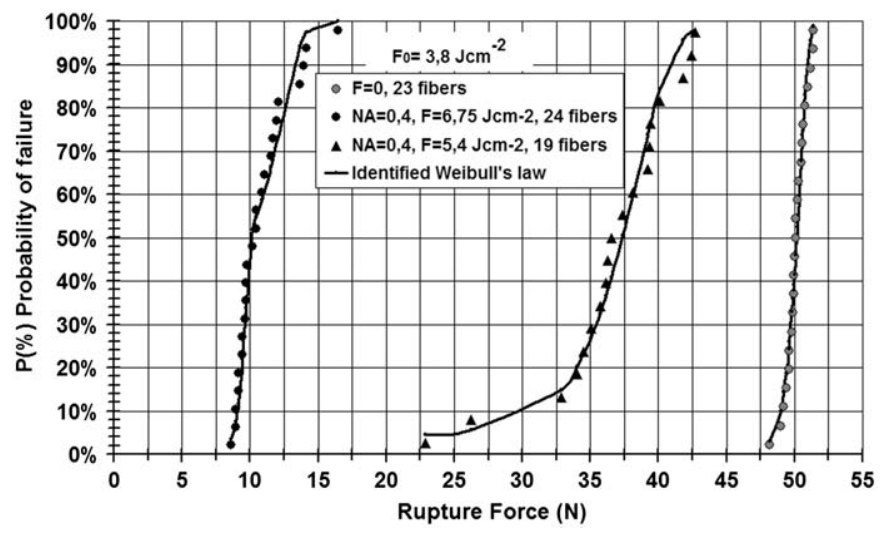

Fig. 17. Failure probability $P(\%)$ of optical fibers as a function of the failure force for two different laser fluences $\left(F=0,5.4 \mathrm{~J} \mathrm{~cm}^{-2}\right.$ and $\left.6.75 \mathrm{~J} \mathrm{~cm}^{-2}\right)$.

fluence, at least for $N A=0.8$, from about 200 to $1020 \mathrm{~nm}$ in the fluence range of $4-18 \mathrm{~J} \mathrm{~cm}^{-2}$. As a good approximation, taking $z^{\prime}=z_{0}$ in the relations (17) and (18) $\left(0.25 \mu \mathrm{m}<z^{\prime}<1.6 \mu \mathrm{m}\right.$ in Fig. $\left.16, z_{0}=0.68 \mu \mathrm{m}\right)$, the evolution as a function of the fluence of the depth $h_{3}$ of the self-focusing craters reported in Fig. 16 $(\mathrm{NA}=0.8)$ is given by

$h_{3}=\frac{2 \delta n_{0} z_{0}}{1+n_{0}^{2} \sqrt{\frac{F}{F_{c}}-1}}$ with $\delta=0.56$ and $F_{c}=3.8 \mathrm{~J} \mathrm{~cm}^{-2}$.

The good reproducibility of these different measurements from one specimen to another one (Spec. 1-6, Figs. 2a,b, 10a,b, 11 and 12) has to be pointed out.

\section{Example of application}

As a first example of mechanical application (sensor for structural composite monitoring), Fig. 17 shows how, knowing the geometries and the distribution of the nano-craters structured on the surface of optical fibers, it is possible to control the rupture strength of these fibers. In this representation, for two different fluences ( $F=0$ : undamaged fibers, $F=5.4$ and $6.75 \mathrm{~J} \mathrm{~cm}^{-2}$ for $\mathrm{NA}=0.4$ ), the failure probability distribution $P(\%)$ is plotted as a function of the rupture force. Note that for the silica of the fiber the fluence threshold is equal to $3.8 \mathrm{~J} \mathrm{~cm}^{-2}$. So, the two classical Weibull's parameters, $m_{0}$ (exponent of the Weibull law) and $\sigma_{0}$ (Weibull's scaling stress) are adjustable thanks to femtosecond laser nano-machining. A complete study of this application, rupture strength of optical fibers as a function of the distribution and the morphologies of the craters will be presented in a further paper.

\section{Conclusions}

The evolution in morphology of nano-craters drilled in borosilicate glass by single laser shots near ablation threshold has been studied by atomic force microscopy and scanning electron microscopy. The influence of the fluence $(3<F<18 \mathrm{~J}$ $\mathrm{cm}^{-2}$ ) and the numerical aperture (NA=0.4 and 0.8) through the focal region was systematically investigated in the case of strong geometrical confinement: nanometric scale in all spatial dimensions. Nano-craters down to less than $100 \mathrm{~nm}$ in depth by $400 \mathrm{~nm}$ in diameter were successfully drilled and imaged. The secondary craters, due to self-focusing of the beam, present a big aspect ratio of the order to 5 when $F>8 \mathrm{~J} \mathrm{~cm}^{-2}$.
The dimensions of the principal and of the secondary (selffocusing) craters, and of the rim have been studied in detail. Different relationships have been proposed for the evolutions of the depths and of the different diameters of the craters as a function of the position of the specimen surface through the beam-material interaction region $\Delta Z$.

This work opens new challenges on light-matter interaction modeling on the nano-scale and new perspectives on ultrafast nano-machining. As an example of mechanical application, the principle of an optical fiber sensor for structural composite monitoring has briefly been presented.

\section{Acknowledgements}

The authors thank the Conseil Regional de Franche Comté, France, for funding, R. Salut for his assistance for the SEM/FIB procedure, P.A. Lacourt and L. Furfaro for the setting of the femtosecond laser.

\section{References}

[1] Gattass RR, Mazur E. Femtosecond laser micromachining in transparent materials. Nat Photon 2008;2(4):219-25.

[2] Yang W, Kazansky PG, Svirko YP. Non-reciprocal ultrafast laser writing. Nat Photon 2008;2(2):99-104.

[3] Lenzner M, Kruger J, Sartania S, Cheng Z, Spielmann C, Marron G, et al. Femtosecond optical breakdown in dielectrics. Phys Rev Lett 1998;80(18): 4076-9.

[4] Joglekar AP, Liu H, Spooner GJ, Meyhofer E, Mourou G, Hunt AJ. A study of the deterministic character of optical damage by femtosecond laser pulses and application to nanomachining. Appl Phys B Lasers Opt 2003;77(1):25-30.

[5] Chimmalgi A, Choi TY, Grigoropoulos CP, Komvopoulous CP. Femtosecond laser aperturless near-field nanomachining of metals assisted by scanning probe microscopy. Appl Phys Lett 2003;82(8):1146-9.

[6] Pronko PP, Dutta SK, Squier J, Rudd JV, Du D, Mourou G. Machining of submicron holes using a femtosecond laser at $800 \mathrm{~nm}$. Opt Commun 1995; 114(1-2):106-10.

[7] Itho K, Watanabe W. Toward nano and microprocessing in glass with femtosecond laser pulses. RIKEN Rev 2003;50:90-4.

[8] Koch J, Korte F, Fallnich C, Ostendorf A, Chichkov BN. Direct-write subwave length structuring with femtosecond laser pulses. Opt Eng 2005;44(5): 051103.

[9] Mao SS, Quere F, Guizard S, Mao X, Russo RE, Petite G, et al. Dynamics of femtosecond laser interactions with dielectrics. Appl Phys A Mat Sci Process 2004;79(7):1695-709.

[10] Ashkenasi D, Muller G, Rosenfeld A, Stoian R, Hertel IV, Bulgakova NM, et al Fundamentals and advantages of ultra fast micro-structuring of transparent materials. Appl Phys A Mat Sci Process 2003;77(2):223-8.

[11] Jia TQ Xu ZZ, Li RX, Feng DH, Li XX, Cheng CF, et al. Mechanisms in femtosecond laser ablation in fused silica. J Appl Phys 2004;95(9):5166-71.

[12] Zhigilei LV. Dynamics of the plume formation and parameters of the ejected clusters in short-pulse laser ablation. Appl Phys A Mat Sci Process 2003;76(3):339-50.

[13] Petrov GM, Davis J. Interaction of intense ultra-short laser pulses with dielectrics. J Phys B At Mol Opt Phys 2008;41(2):025601.

[14] Guizard S, Semerok A, Gaudin J, Hashida A, Martin P, Quere F. Femtosecond laser ablation of transparent dielectrics: measurement and modelisation of crater profiles. Appl Surf Sci 2002;186(1-4):364-8.

[15] Englert L, Rethfeld B, Haag L, Wollenhaupt M, Sarpe-Tudoran C, Baumert T. Control of ionization processes in high band gap material via tailored femtosecond pulses. Opt Express 2007;15(26):17855-62.

[16] Bouilly D, Perez D, Lewis LJ. Damage in materials following ablation by ultrashort laser pulses. A molecular-dynamics study. Phys Rev B 2007;76:184119.

[17] Bennett TD, Krajrobich DJ, Grigoropoulous CP, Baumgart P, Tam AC. Maragoni mechanism in pulsed laser texturing of magnetic disk substrates. J Heat Transfer 1997;119(3):589-96.

[18] Nolte S, Momma C, Kamlage G, Ostendorf A, Fallnich C, Von Alvensleben F, et al. Polarization effects in ultra short-pulse laser drilling. Appl Phys A Mat Sci Process 1999;68(5):563-7.

[19] Nielsen CS, Balling P. Deep drilling of metals with ultrashort laser pulses: a two stage process. J Appl Phys 2006;99(9):093101.

[20] Little DJ, Ams M, Dekker P, Marshall GD, Dawes JM, Withford MJ. Femtosecond laser modification of fused silica: the effect of writing polarization on Si-0 ring structure. Opt Express 2008;16(24):20029-37.

[21] Kazansky PG, Yang W, Bricchi E, Bovatsek J, Arai A, Shimotsuma Y, et al. Quill writing with ultrashort light pulses in transparent materials. Appl Phys Lett 2007;90(15):151120. 
[22] Gamaly EG, Rode AV, Luther-Davies B, Tikhonchuk VT. Ablation of solids by femtosecond lasers: ablation mechanism and ablation thresholds for metals and dielectrics. Phys Plasmas 2002;9(3):949-57.

[23] Xu S, Qiu J, Jia T, Li C, Sun H, Xu Z. Femtosecond laser ablation of crystals $\mathrm{SiO}_{2}$ and YAG. Opt Commun 2007;274:163-6.

[24] Jia TQ, Xu ZZ, Li XX, Li X, Shuai B, Zhao FL. Microscopic mechanisms of ablation and micromachining of dielectrics by using femtosecond lasers. Appl Phys Lett 2003;82(24):4382-4.
[25] White YV, Li X, Sikorski Z, Davis LM, Hofmeister W. Single-pulse ultrafast laser machining of high aspect nano-holes at the surface of $\mathrm{SiO}_{2}$. Opt Express 2008;16(19):14411-20.

[26] Hwang DJ, Grigoropoulos CP, Choi TY. Efficiency of silicon micromachining by femtosecond laser pulses in ambient air. J Appl Phys 2006;99:083101.

[27] Sudrie L, Franco M, Prade B, Mysyrowicz A. Study of damage in fused silica induced by ultra-short IR laser pulses. Opt Commun 2001;191: 333-339. 